\title{
A Reliable Subcasting Protocol for Wireless Environments
}

\author{
Djamel H. Sadok, Carlos de M. Cordeiro and Judith Kelner \\ Centro de Informática, Universidade Federal de Pernambuco, Caixa Postal: 7851 \\ Cidade Universitária, Recife, PE, Brasil \\ \{jamel, cmc, jk\}@di.ufpe.br
}

\begin{abstract}
This paper presents an end-to-end reliable multicast protocol for use in environments with wireless access. It divides a multicast tree into sub-trees where subcasting within these smaller regions is applied using a tree of retransmission servers (RSs). RM2 is receiver oriented [1] in that the transmitter does not need to know its receivers, hence offering better scalability. The Internet Group Management Protocol (IGMP) is used manage group membership whereas the IETF's Mobile IP offers support to user mobility and a care-of address (temporary IP address). Each RS has a retransmission subcast address shared by its members and which may be dynamically configured using IETF's MADCAP (Multicast Address Dynamic Client Allocation Protocol) [8]. Most importantly, RM2 uses a dynamic retransmission strategy to switch between multicast and unicast retransmission modes according to the extra load generated in the network as well as the wireless interfaces by packet retransmissions. It is shown through both analytical modeling and simulation that RM2's dynamic adaptation is not only important but necessary when considering mobile access.
\end{abstract}

\section{Introduction: Multicast in Mobile Environments}

The IETF defines two approaches to multicast at the IP level, namely bi-directional tunneling and remote subscription. Other techniques for unreliable multicast have also been adopted in [4, 5, 6, 7]. The solution adopted in [4], and later on refined in [5], has scalability problems and assumes that group membership is static, which is hardly true when considering mobile environments. [6] and [7] deals with problems such as tunnel convergence, but does not deal with packet loss and performance issues.

Commonly used reliable protocols include the scalable reliable multicast (SRM) [2] and the reliable multicast transport protocol (RMTP) [3]. On the one hand, SRM is based on an application level framework where it is the application's responsibility to guarantee packet sequencing. This protocol is compared to RM2 using simulation later in the paper. On the other hand, RMTP defines a hierarchy of designated routers (DRs), a concept also used in RM2. Although each DR is responsible for handling error recovery within a region of the multicast tree, it does not say whether this is done in multicast or unicast and, therefore, does not concern itself with the control of retransmission overhead. This is a serious drawback when considering the emerging mobile environments. 
In this paper, a new reliable multicast protocol, called RM2 (Reliable Mobile Multicast), tailored for mobile environments is presented.

\section{Specification of the RM2 Protocol}

The role of RM2 is to take a stream of packets generated by an application and deliver it to all mobile as well as fixed hosts interested in receiving it in a reliable and optimized way. Furthermore, RM2 guaranties sequential packet delivery with no packet loss to all its multicast members.

RM2 assumes that the network is formed of multicast routers and that cells are big enough to allow users to join and leave multicast groups. The RSs (Retransmission Servers) perform selective retransmissions on the basis of feedback in the form of negative acknowledgements they receive. RM2 dynamically establishes the subcasting regions while taking into account retransmission costs.

\subsection{The Role of a Retransmission Server}

A multicast sender must first divide a multicast message into smaller fixed size packets (except the last one). To each one of these packets, RM2 associates a sequence number $\left(\mathbf{n}_{\mathbf{s e q}}\right)$. In order to guarantee end-to-end reliability, the receivers are required to send NACKs pointing out which packets they want to be retransmitted. In other words, RM2 implements selective packet retransmission. A NACK contains a sequence number $\mathrm{N}$ and bitmap $\mathrm{B}$. $\mathrm{N}$ indicates that all packets with sequence number less than $\mathrm{N}$ have been correctly received by a given receiver. The bitmap $\mathrm{B}$, on the other hand, shows which packets have actually been received. Consider the example where the ACK contains $\mathrm{N}=22$ and $\mathrm{B}=01111101$. In this case, the receiver wishes to indicate that it has correctly received all multicast packets with a sequence number less than 22 and that it is requesting the retransmission of packets 22 and 28 as indicated by the two 0s present in the bitmap B.

Sending NACKs to a multicast sender could lead to overwhelming it and causing an NACK implosion and even network congestion especially near the multicast source subnet. Therefore, RM2 divides the multicast network into hierarchical regions, where each one of these is controlled by a retransmission server (RS). This is responsible for gathering and processing NACKs from its region and for the retransmission of packets as requested by some receivers. Figs. 1(a) and 1(b) illustrate this concept. RM2 assigns RS functionality to fixed hosts selected on a network topology basis. The selection is subject to analysis in the following text.

The RSs are centric to RM2 support for reliable multicast sessions. Multicast packets are cached within the RSs buffers. The RSs are also responsible for the combination of NACKs received from lower RS hierarchies and hosts within their regions and responding to these when possible. If not, the retransmission request may be passed on to higher levels of RSs. Note that the separation between multicast routers and RSs is purposely done. It has the benefit of freeing router resources to the handling of multicast packets. RSs, on the hand, are better represented by hosts since they may need to use large buffering to keep copies of multicast packets for possible retransmission. 


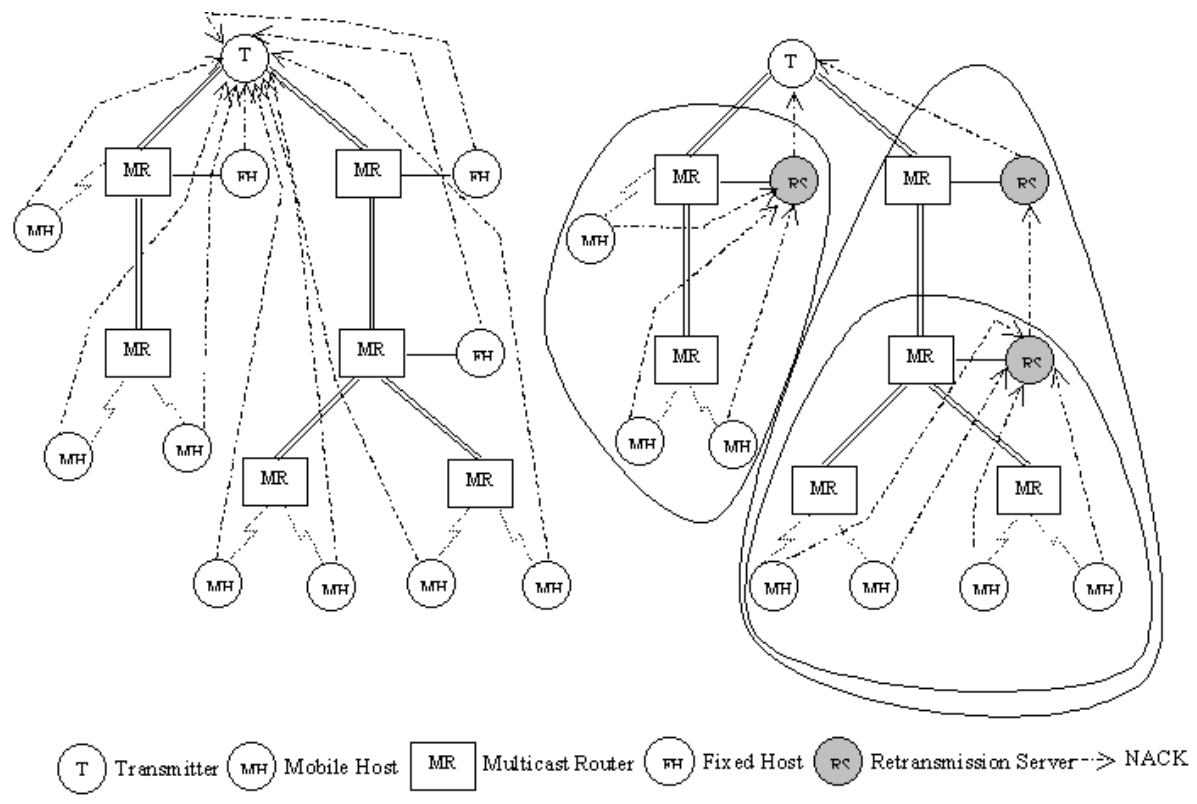

Fig. 1(a). Receivers sending NACKs to the multicast transmitter

Fig. 1(b). RS NACK processing

\subsection{Establishing the RS Hierarchy}

Core to the RM2 protocol is the establishment of a minimum cost spanning tree of RSs. The static selection of RSs in a WAN is on the basis of network topology. However, a multicast receiver selects dynamically its RS. Initially when there are no users in group a $\mathrm{G}$, the RSs transmit a CFG_RS packet for $\mathrm{G}$ at each time interval $\mathrm{T}_{\mathrm{RS}}$ in order to:

- Advertise its status as an RS to all potential members of a group;

- Define transmission costs where each CFG_RS packet contains a field with the cost of reaching the transmitter. This cost is null when the RS is not currently receiving data for $\mathrm{G}$ or that it is the actual transmitter. An example of a useful cost metric used in the simulation is the number of hops.

As soon as hosts join the multicast group they start receiving, in addition to multicast data packets, CFG_RS configuration packets. This way a host may be able to select the one that it is nearest to it on the basis on the cost information. The host then sends a REG_HOST to a suitable RS and sets a timer with a $2 * \mathrm{~T}_{\mathrm{RS}}$ value. The REG_HOST message has the following information: 
- HostType: telling its RS whether it is a fixed or mobile host;

- Group ID: of the multicast group it is currently joining;

On receipt of a REG_HOST message a RS invokes the following actions:

- The RS updates its fixed/mobile host counters within its region and registers the hosts' unicast address;

- In the event that this is the first host to enter a multicast group at this RS, it dynamically allocates a D (multicast type) address, for example G1, using a protocol such as MADCAP [8]. This is the retransmission address for all receivers at this RS. Next the RS sends a RETR_ADDR packet telling its new host which multicast retransmission address its has to listen to. From now on, a new group G1 with a subcasting address has been established at this RS;

- Based on the number of retransmission requests a RS receives within a region, it may retransmit using either unicast or subcasting. This flexibility is a clear advantage over other protocols such as RMTP and SRM. Details of this mechanism are presented later in the paper. With the insertion of new hosts, new regions with separate RSs are formed. Each new RS performs the same procedure as a host in order to get a retransmission feed from a higher level RS. RM2 uses the Djikistra algorithm to establish the spanning RS tree where hop count is cost metric.

\subsection{Support for Fixed Receivers}

At the network level IGMP is used for group management. Existing multicast routing protocols such as the distance vector multicast routing protocol (DVMRP), protocol independent multicast (PIM) and multicast OSPF (MOSPF) may be used in conjunction with RM2 for routing multicast packets. A RS distinguishes between mobile and fixed hosts using the HostType field present in the REG_HOST registration message. Similarly, a host may leave a retransmission subcast group at a RS by sending it a LEAVE_GROUP message. The RS needs to keep track of the number of hosts in its region and should remove its link to the upper RS when it has no members to serve.

\subsection{Support for Mobile Receivers}

Mobile devices are somehow more delicate to handle at the RS. MHs send a normal IGMP join message to a multicast router (MR) using the care-of address from a foreign network (FN) obtained through the use of the IETF's mobile IP. The MR uses normal procedure to include itself in the IP multicast tree. The $\mathrm{MH}$ then sends a REG_HOST to its closest RS with the HostType field set to mobile. The following situations may happen as a result of host mobility:

- A MH sends an IGMP leave group message to its current MR before performing handoff. The MR may then check through a new IGMP query message to see if there are still multicast host members in its subtree. If not, 
it would remove itself from the multicast tree. Furthermore, the $\mathrm{MH}$ also sends a RM2 LEAVE_GROUP message to its respective RS in order to be removed from the retransmission subcast list;

- A second situation rises when the $\mathrm{MH}$ is unable to leave the group before handoff is complete or that the "leave" message is lost. Therefore, the MR may only know if there are hosts left at its subtree only after it sends a new IGMP membership query message. Since the IGMP leave message may itself be lost be lost, a registration timeout is associated to each MH. The RS relies on NACKs sent by MHs to know which of these remain active in its region. An interesting scenario emerges when a $\mathrm{MH}$ performs a handoff and moves to a new network (at another FA). This leads to packet loss due to the fact that the $\mathrm{MH}$ is unable to receive multicast packets when it is in between FAs. At reconnection, the MH must send an RM2 REQUEST message to start receiving from where it left the multicast transmission before the handoff.

\subsection{Support for Mobile Transmitters}

Reconfiguring the entire multicast tree each time a sending $\mathrm{MH}$ moves to a new $\mathrm{FN}$ is costly. Another problem results from the sending of NACKs to a FN where the MH no longer is. RM2 adopts two approaches to deal with these problems:

- When the $\mathrm{MH}$ is within its home network, it performs a link level multicast. The HA then forwards these downstream;

- If the MH is visiting a FN, it tunnels packets to its HA which then forwards these using local links and WAN interfaces. In other words, the HA in this case performs the role of a gateway. For performance and scalability reasons, RM2 limits the use of IP tunnels when there is a multicast mobile source transmitting.

\subsection{RM2 Error Recovery}

NACKs are used by hosts to signal lost and corrupt packets. RM2 adopts a similar approach to RMTP in that it collects NACKs per packet within a queue during a specific time interval and then retransmits the requested packets. This scheme clearly needs further tuning in the case of mobile hosts, especially considering that the requesting mobile hosts may have moved on to new FNs since they sent NACKs when dealing with high mobility users for example. RM2 adopts a dynamic retransmission technique at the RS level as shown in the following two scenarios:

- High error rate: representing environment with a high number of mobile users with wireless access;

- Low error rates: representing situations with a relatively low mobile to fixed users ratio;

A RS continuously monitors the number of fixed and mobile users and their NACKs. It is shown when describing the analytical model for RM2 that there are two 
main mathematical restrictions that control the adaptation of the retransmission algorithm that decides whether retransmitted packets at a RS are sent to all group members via multicast (more specifically subcast) or that they are sent via unicast to all those hosts that requested them. When the number of NACKs is relatively low, unicast is used at the RS to retransmit packets. RM2 monitors the network load, as shown in the analytical model, and should this becomes relatively high, it would then switch to multicasting retransmission packets. However, it is important that RM2 does not attempt to reduce network load at the detriment of low speed wireless interfaces. Indeed, RM2 only uses multicast as long as the wireless channel occupation remains bellow a threshold. Between overloading the fixed network and the wireless links, RM2 chooses to limit the traffic on the latter. There are, however, situations where, even when unicast is used at the RS, the wireless interface may see too many retransmission packets. The RS may then send, on behalf of its MHs, a REDUCE_FLOW message to the multicast source. Note that it is not mandatory for a transmitter to comply with this message. Table 1 shows a summary of RM2 messages.

Table 1. RM2 messages

\begin{tabular}{|c|c|c|c|}
\hline Message & Originator & Receiver & Description \\
\hline DATA & Transmitter & Receivers \& RSs & Data Packet \\
\hline REQUEST & Receivers \& RSs & RSs & $\begin{array}{l}\text { Requests the } \\
\text { retransmission of lost } \\
\text { packets }\end{array}$ \\
\hline REPAIR & RSs & Receivers \& RSs & $\begin{array}{l}\text { Retransmission of lost } \\
\text { packets }\end{array}$ \\
\hline CFG_RS & Transmitter \& RSs & Receivers \& RSs & $\begin{array}{l}\text { Building RS tree and } \\
\text { establishing } \\
\text { regions }\end{array}$ \\
\hline REG_HOST & Receivers and RSs & RSs & Host registration \\
\hline RETR_ADDR & RSs & Receivers \& RSs & $\begin{array}{lr}\text { Informs a region's } \\
\text { retransmission } & \text { subcast } \\
\text { address } & \end{array}$ \\
\hline LEAVE_GROUP & Receivers \& RSs & RSs & $\begin{array}{l}\text { Indicates that a host is } \\
\text { leaving a group or } \\
\text { simply doing a handoff. }\end{array}$ \\
\hline REDUCE_FLOW & RSs & RSs & Flow Control \\
\hline
\end{tabular}

\section{Modeling Packet Retransmission}

A $n$-ary network topology with a depth $h$ is considered with equal fixed link costs and where retransmission requests are assumed to be uniformly distributed. If $P S$ denotes the packet size, then equations (1) and (2) give the unicast and multicast retransmission costs to $K$ receivers respectively. 


$$
\begin{aligned}
C_{U}(K, h, P S) & =K \times h \times P S . \\
C_{M}(n, h, P S) & =n \frac{n^{h}-1}{n-1} P S, n>1 .
\end{aligned}
$$
(3):

The tree depth $h$ depends is related to the total number of hosts $\left(\mathrm{N}_{\mathrm{T}}\right)$ as shown in

$$
h\left(N_{T}, n\right)=\log _{n}\left(\frac{N_{T}(n-1)}{n}+1\right) .
$$

Define $\mathrm{P}\left(\mathrm{Xf}_{\mathrm{i}, \mathrm{j}}\right)$ and $\mathrm{P}\left(\mathrm{Xm}_{\mathrm{i}, \mathrm{j}}\right)$ as the probability that a fixed/mobile host $i$ requests a retransmission of packet $\mathrm{j}$. They are respectively given by:

$$
\begin{aligned}
& \mathrm{P}\left(\mathrm{Xf}_{\mathrm{i}, \mathrm{j}}\right)=1-\left(1-\mathrm{P}\left(\mathrm{E}_{\mathrm{F}}\right)\right)^{\mathrm{h}} \\
& \mathrm{P}\left(\mathrm{Xm}_{\mathrm{i}, \mathrm{j}}\right)=1-\left(1-\mathrm{P}\left(\mathrm{E}_{\mathrm{F}}\right)\right)^{\mathrm{h}-1}\left(1-\mathrm{P}\left(\mathrm{E}_{\mathrm{M}}\right)\right) .
\end{aligned}
$$

where $\mathrm{E}_{\mathrm{F}}$ and $\mathrm{E}_{\mathrm{M}}$ are packet error rates for fixed and wireless links.

Let $\mathrm{P}\left(\mathrm{Xf}_{\mathrm{i}, \mathrm{j}}\right)$ and $\mathrm{P}\left(\mathrm{Xm}_{\mathrm{i}, \mathrm{j}}\right)$ be the probabilities that a fixed or mobile host i requests the retransmission of packet $\mathrm{j}$ respectively. Furthermore, let $\mathrm{P}_{\mathrm{i}}\left(\mathrm{Y}=\mathrm{K}_{\mathrm{F}}, \mathrm{Z}=\mathrm{K}_{\mathrm{M}}\right)$ represent the probability of having exactly $\mathrm{K}_{\mathrm{F}}$ and $\mathrm{K}_{\mathrm{M}}$ retransmission requests of a given packet $\mathrm{i}$ for both fixed and mobile hosts, while $\mathrm{N}_{\mathrm{F}}$ is the total number of fixed hosts and $\mathrm{N}_{\mathrm{M}}$ the total number of mobile hosts. Since retransmission request events are independent and that $\mathrm{P}\left(\mathrm{Xf}_{\mathrm{i}, \mathrm{j}}\right)$ and $\mathrm{P}\left(\mathrm{Xm}_{\mathrm{i}, \mathrm{i}}\right)$ remain practically constant during the experiment, $\mathrm{P}_{\mathrm{i}}(\mathrm{Y}, \mathrm{Z})$ may be modeled as a binomial distribution. Therefore, we have:

for fixed hosts, and

$$
P_{i}\left(Y=K_{f}\right)=\left(\begin{array}{l}
N_{F} \\
K_{f}
\end{array}\right) P\left(X f_{i, j}\right)^{K_{f}}\left(1-P\left(X f_{i, j}\right)\right)^{N_{F}-K_{f}} .
$$

for mobile hosts.

$$
P_{i}\left(Z=K_{m}\right)=\left(\begin{array}{c}
N_{M} \\
K_{m}
\end{array}\right) P\left(X m_{i, j}\right)^{K_{m}}\left(1-P\left(X m_{i, j}\right)\right)^{N_{M}-K_{m}} .
$$

Through consecutive mathematical refinements of equations (4) and (5), we obtain equations (6) and (7) as representing packet retransmission in both unicast and multicast modes:

$$
\begin{gathered}
E\left(C R_{U}\right)=h P S\left(N_{F} P\left(X f_{i, j}\right)\left(1-P\left(X f_{i, j}\right)\right)+\left(N_{F} P\left(X f_{i, j}\right)\right)^{2}+\right. \\
\left.N_{M} P\left(X m_{i, j}\right)\left(1-P\left(X m_{i, j}\right)\right)+\left(N_{M} P\left(X m_{i, j}\right)\right)^{2}\right) . \\
E\left(C R_{M}\right)=n \frac{n^{h}-1}{n-1} P S\left(N_{F} P\left(X f_{i, j}\right)+N_{M} P\left(X m_{i, j}\right)\right) .
\end{gathered}
$$

Fig. 2 presents the network load behavior as the number of retransmission requests arriving at the transmitter increases (equations (i) and (ii)). We see that, as the number 
of requests grows, the overload generated by the retransmitted packets depends on the retransmission mode (unicast or multicast). Ideally, RM2 should change into multi-

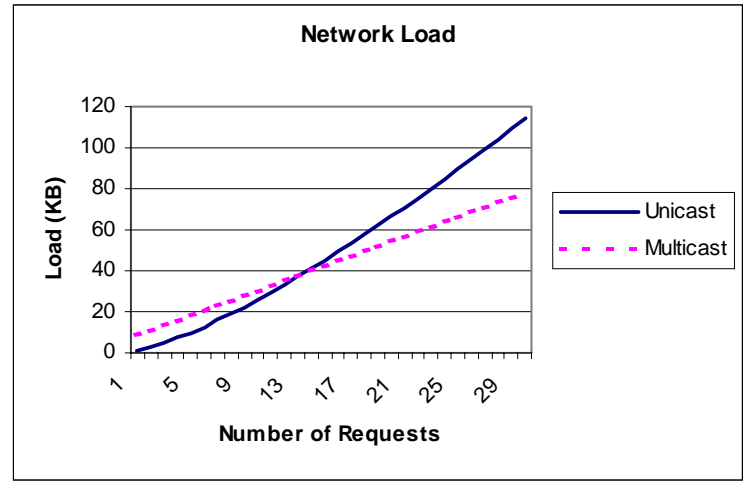

Fig. 2. Network Load

cast retransmission when the two curves intersect as shown in fig. 2. Table 2 shows some of parameters used for this analysis. Through the variation of the ratio mobile/fixed hosts, we show that the effect of mobile hosts is far more important on retransmission load and that the initial unicast retransmission scheme cannot be maintained when there is a large network.

Table 2. Some Parameters for the Analytical Evaluation

\begin{tabular}{ll}
\hline \multicolumn{1}{c}{ Parameter } & \multicolumn{1}{c}{ Value } \\
\hline $\mathrm{P}\left(\mathrm{E}_{\mathrm{F}}\right)$ & $10^{-9}$ \\
$\mathrm{P}\left(\mathrm{E}_{\mathrm{M}}\right)$ & $10^{-3}$ \\
$\mathrm{n}$-arity & 2 \\
$\mathrm{PS}($ Packet Size) & $1 \mathrm{~KB}$ \\
\hline
\end{tabular}

Actually, the RM2 retransmission mechanism takes into account two restrictions: R1 and R2. R1 guaranties that the network load does not exceed an established threshold $p$ whereas $\mathrm{R} 2$ ensures that wireless retransmission channel utilization is not overwhelmed by multicast retransmitted packets. R1 is given below (equation 8):

$$
\alpha . E\left(C R_{U}\right) \leq p
$$

Where $\alpha$ :

$$
\alpha=\frac{1}{N_{M}} \cdot \frac{N_{M} P\left(E_{M}\right)}{N_{M} P\left(E_{M}\right)+N_{F} P\left(E_{F}\right)}=\frac{P\left(E_{M}\right)}{N_{M} P\left(E_{M}\right)+N_{F} P\left(E_{F}\right)} .
$$

In other words, $\alpha$ represents the average error rate related to fixed and wireless errors as well as the number of both user types. Fig. 3 shows the impact of both fixed $\left(\mathrm{N}_{\mathrm{F}}\right)$ and mobile users $\left(\mathrm{N}_{\mathrm{M}}\right)$ (equations (vi) and (vii)). Through fig. 3, we see that mobile users have a much bigger effect on the load generated by duplicate packets 
than fixed users. Therefore, RM2's retransmission mechanism was specially adapted to deal with mobile users.

$\mathrm{R} 2$, on the other hand, is given by:

$$
E\left(C R_{M}\right) \leq E\left(C R_{U}\right) .
$$

That is to say that RM2 must always check if it is not time to switch from a unicast to multicast retransmission in order to lower the network packet retransmission load.
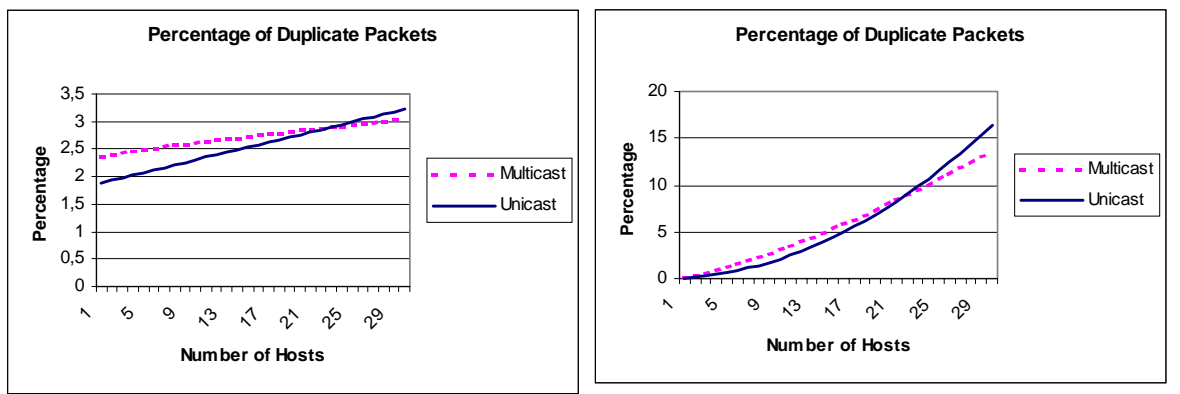

Fig. 3. Impact of Fixed and Mobile hosts

\section{RM2 Simulation}

RM2 was implemented in the U.C. Berkeley/LBNL ns-2 simulator. The source code is available from http://www.di.ufpe.br/ cmc/research/rm2/rm2-source.zip.

Topology. A backbone topology with 7 multicast routers has been used with 2 Mbps links and 10ms delay. The scenario consists of a maximum of 50 users joining a multicast transmission at different levels of the hierarchy. Fixed access is characterized by a $10 \mathrm{Mbps}$ speed and $5 \mathrm{~ms}$ delay, whereas mobile access uses $14 \mathrm{kbps}$ and a 50ms delay. We used ns-2 DVMRP (Distance Vector Multicast Routing Protocol) for building routing tables, IGMP for group management and mobile IP for address allocation.

Transmission. Each simulation experiment starts at time 1 second, and after the bootstrap phase of 8 seconds a CBR (constant bit rate) source starts transmitting 5000 $1 \mathrm{~KB}$ packets at $64 \mathrm{Kbps}$.

Error Model. In RM2's simulation, errors are a result of buffer overflow in routers and transmission packet error rates. Whereas the first one represents the dominant source for Internet packet loss, the second one reflects the error probability inherent to each link, as explained in the analytical model and configured in table 2 . As presented earlier, the analytical model determines that the loss rates receivers experience are obtained by compounding the loss rates on the links from the sender to the receivers. 
Results. The results mainly validate the analytical modeling earlier shown. The network load shown in fig. 4 presents similar tendencies to the one resulting from the analytical model presented fig. 2 .

Similarly, the results show that the impact (see fig. 5) of both fixed and mobile users is in line with the one from the analytical model shown in fig. 3.

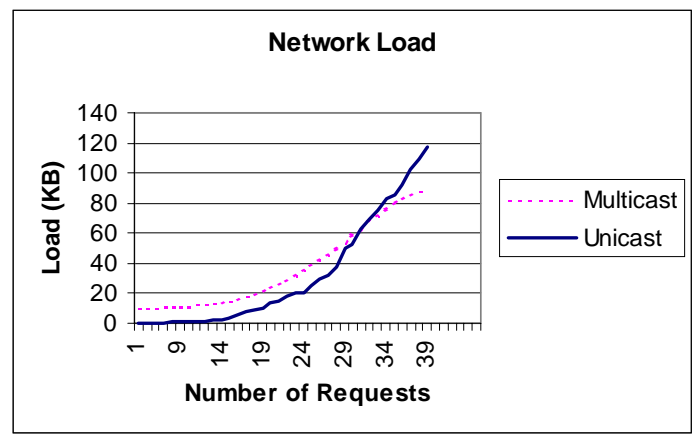

Fig. 4. Simulation of Network Load
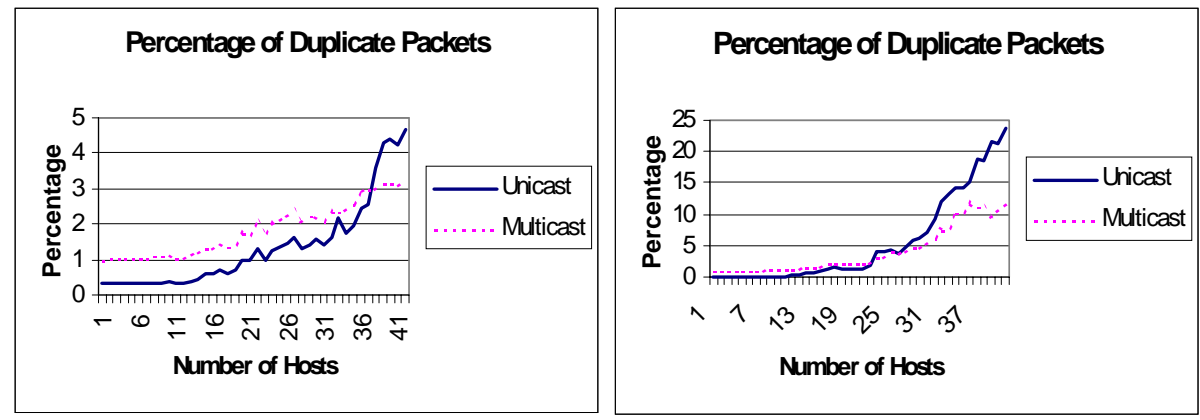

Fig. 5. Impact of Fixed and Mobile Hosts

\section{Comparing RM2 Performance with SRM}

Finally, we compare RM2 and SRM performance under similar loads. SRM has been selected since it is well referenced in the literature and readily available in the ns-2 simulator. We set R1 as a condition for RM2 to ensure that wireless interface may not see more than $20 \%$ of retransmission. Fig. 6 illustrates link utilization during the simulation.

The results are somehow intuitive, SRM generates higher levels of network occupancy since it always retransmits in multicast mode which may of course overload low speed wireless interfaces (in fig. 6, there are times where SRM uses almost $80 \%$ of this capacity). 


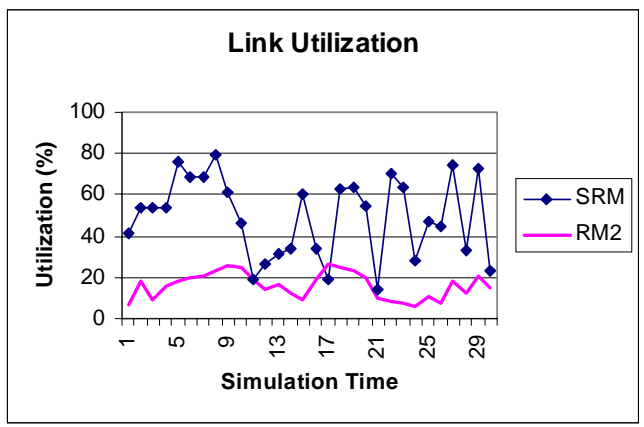

Fig. 6. Utilization of Wireless Links in SRM and RM2
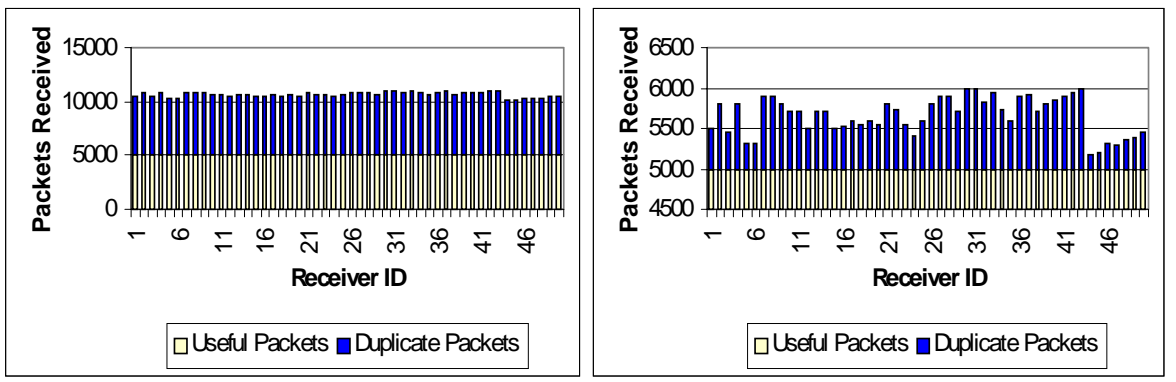

Fig. 7. SRM Duplicate Packets (left) and RM2 Duplicate Packets (right)

Furthermore, SRM's average occupation of wireless interfaces was 50\% compared to a mere $16 \%$ when using RM2. Fig. 7 shows the number of duplicates seen by a host. Since SRM uses multicast for retransmitting packets to all group members, it presents a large overhead (close to 100\%). This is often unacceptable for mobile hosts specially when compared to the overhead of RM2 which is less than $20 \%$ (maintaining restriction R1). Clearly, RM2 retransmission adaptation not only optimizes network traffic load but more importantly spares low speed wireless links.

\section{Conclusions}

This work has presented a new reliable multicast protocol, suitable for use in wireless environments. RM2 defines a hierarchy of retransmission servers which implement subcasting. It was shown through analytical modeling and simulation that the adaptation mechanism reduces multicast traffic due to packet retransmission and saves link utilization at the wireless interface. Finally, through simulation, RM2 efficiency over SRM was shown to be superior. 


\section{References}

1. S. Pingali, D. Towsley, J. Kurose, "A Comparison of Sender-Initiated and Receiver-Initiated Reliable Multicast Protocols", Proc. ACM SIGMETRICS Conf. On Measurement and Modeling of Computer Systems, May 1994

2. S. Floyd, V. Jacobson, S. McCanne, C. Liu, L. Zhang, "A Reliable Multicast Framework for Light-Weight Sessions and Application Level Framing", ACM SIGCOMM'95, Conf. on Applications, Technologies, Architectures and Protocols for Computer Communications, August 1995

3. S. Paul, K. Sabnani, J. Lin, S. Bhattacharrya, "Reliable Multicast Transport Protocol (RMTP)", IEEE Journal on Selected Areas in Communications, April 1997

4. A. Acharya, B. Badrinath, "Delivering Multicast Messages in Networks with Mobile Hosts", Proc. Of the $13^{\text {th }}$ International Conference on Distributed Computer Systems, May 1993

5. A. Acharya, B. Badrinath, "A Framework for the Delivery of Multicast Messages in Networks with Mobile Hosts", Wireless Networks, 1996

6. V. Chikarmane, C. Williamson, R. Bunt. W.Mackrell, "Multicast Support for Mobile Hosts Using Mobile IP: Design Issues and Proposed Architecture", ACM/Baltzer Mobile Networking and Applications, 1997

7. T. Harrison, C. Williamson, R. Bunt. W.Mackrell, "Mobile Multicast (MoM) Protocol: Multicast Support for Mobile Hosts", Department of Computer Science, University of Saskatchewan, Canada

8. MADCAP Protocol, http://www.ietf.org/internet-drafts/draft-ietf-malloc-madcap-07.txt 